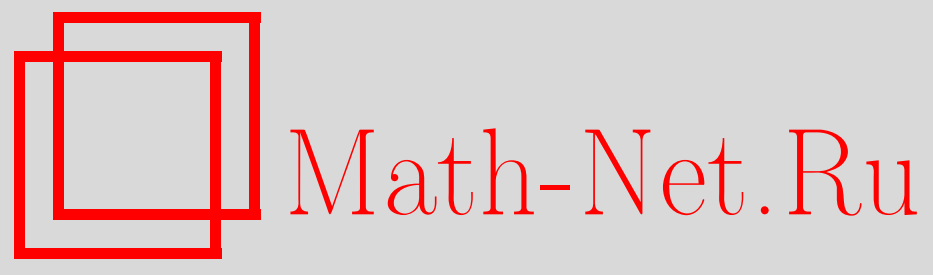

С. Я. Шатских, Усиленный закон больших чисел для схемы серий условных распределений эллиптически контурированных мер, Теория вероятн. и ее примен., 2005, том 50, выпуск 2, 292-311

DOI: https://doi.org/10.4213/tvp108

Использование Общероссийского математического портала Math-Net.Ru подразумевает, что вы прочитали и согласны с пользовательским соглашением

http://www.mathnet.ru/rus/agreement

Параметры загрузки:

IP: 52.6 .47 .48

26 апреля 2023 г., 18:24:53

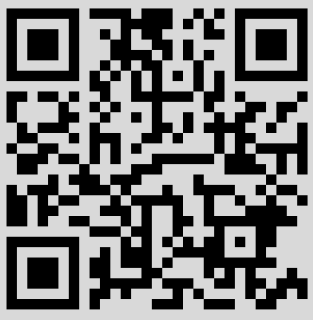




\section{УСИЛЕННЫЙ ЗАКОН БОЛЬШИХ ЧИСЕЛ ДЛЯ СХЕМЫ СЕРИЙ УСЛОВНЫХ РАСПРЕДЕЛЕНИЙ ЭЛЛИПТИЧЕСКИ КОНТУРИРОВАННЫХ МЕР ${ }^{1)}$}

Работа посвящена изучению свойств условных распределений устойчивых эллиптически контурированных мер, заданных на вещественном гильбертовом пространстве. Рассматриваются проекции меры на возрастающую последовательность конечномерных линейных пространств, порожденных начальными отрезками ортонормированного базиса. Показано, что асимптотические свойства условных распределений, соответствующих таким проекциям меры, зависят от выбора базиса. Получены достаточные условия выбора ортонормированного базиса гильбертова пространства, при выполнении которых семейство серий условных распределений, в определенном смысле, удовлетворяет усиленному закону больших чисел.

Ключевые слова и фразы: устойчивые эллиптически контурированные меры, условные распределения, ортонормированные базисы, представление Шенберга, эквивалентные гауссовские меры, правильные операторы, сходимость почти наверное.

1. Введение и формулировка основных результатов. Рассмотрим вещественное сепарабельное гильбертово пространство $\mathbf{H}$ со скалярным произведением $\langle\cdot, \cdot\rangle$ и борелевской $\sigma$-алгеброй $\mathscr{B}(\mathbf{H})$. Пусть $\mu_{\alpha}\{\cdot ; B\}$ - устойчивая эллиптически контурированная мера на $\{\mathbf{H}, \mathscr{B}(\mathbf{H})\}(\alpha \in] 0,2])$ с характеристическим функционалом

$$
\Psi_{\alpha}(y)=\exp \left\{-\langle B y, y\rangle^{\alpha / 2}\right\}, \quad y \in \mathbf{H},
$$

где $B: \mathbf{H} \rightarrow \mathbf{H}$ - линейный самосопряженный положительно определенный ядерный оператор, собственные векторы которого $\left\{e_{n}\right\}$ образуют ортонормированный базис (о.н.б.) в пространстве $\mathbf{H}$.

Выберем $\left\{f_{k}\right\}$ - произвольный о.н.б. в гильбертовом пространстве $\mathbf{H}$ и рассмотрим функцию распределения проекции меры $\mu_{\alpha}\{\cdot ; B\}$ на линейную оболочку векторов $f_{1}, \ldots, f_{n}$ :

$$
F_{1 \ldots n}^{(\alpha)}\left(x_{1}, \ldots, x_{n}\right):=\mu_{\alpha}\left\{h \in \mathbf{H}:\left\langle h, f_{1}\right\rangle \leqslant x_{1}, \ldots,\left\langle h, f_{n}\right\rangle \leqslant x_{n} ; B\right\} .
$$

* Самарский государственный университет, ул. Академика Павлова, 1, 443011 Самара, Россия; e-mail: shatskih@ssu.samara.ru

1) Работа выполнена при поддержке гранта Президента РФ НШ-1758.2003.1. 
Кроме того, рассмотрим $F_{i \mid 1 \ldots \hat{\imath} \ldots n}^{(\alpha)}\left(x_{i} \mid x_{1}, \ldots, \widehat{x_{i}}, \ldots, x_{n}\right)-$ условную функцию распределения случайной величины $\left\langle h, f_{i}\right\rangle$ относительно системы $\left\langle h, f_{1}\right\rangle, \ldots,\left\langle\widehat{h, f}_{i}\right\rangle, \ldots,\left\langle h, f_{n}\right\rangle$; - - знак пропуска элемента.

В связи с введенными функциями $F_{i \mid 1 \ldots \hat{\imath} . . . n}^{(\alpha)}$ возникает вопрос: в какой степени их асимптотические свойства $(n \rightarrow \infty)$ зависят от выбора о.н.б. $\left\{f_{k}\right\}$ ?

Перейдем к формулировкам основных результатов. Для этого, используя о.н.б. $\left\{f_{k}\right\}$, введем семейство ортопроекторов

$$
\pi_{m}: \mathbf{H} \rightarrow \mathbf{H}_{m}
$$

где подпространства $\mathbf{H}_{m}:=\operatorname{span}\left\{f_{1}, \ldots, f_{m}\right\}, m=1,2, \ldots$

Теорема 1. Если ортонормированный базис $\left\{f_{k}\right\}$ обладает свойством

$$
\sum_{n=1}^{\infty}\left[\frac{1}{n^{2}} \sum_{i=1}^{n} \sum_{j=1}^{n} \frac{\left\langle\left(\pi_{n} B \pi_{n}\right)^{-1} f_{i}, f_{j}\right\rangle^{2}}{\left\langle\left(\pi_{n} B \pi_{n}\right)^{-1} f_{i}, f_{i}\right\rangle\left\langle\left(\pi_{n} B \pi_{n}\right)^{-1} f_{j}, f_{j}\right\rangle}\right]^{2}<+\infty
$$

то для любого $\alpha \in] 0,2]$ для $\mu_{\alpha}\{\cdot ; B\}$-почти всех $h \in \mathbf{H}$

$$
\lim _{n \rightarrow \infty} \frac{1}{n} \sum_{i=1}^{n}\left[\Phi^{-1}\left(F_{i \mid 1 \ldots \hat{\imath} \ldots n}^{(\alpha)}\left(h_{i} \mid h_{1}, \ldots, \widehat{h_{i}}, \ldots, h_{n}\right)\right)\right]^{2}=1
$$

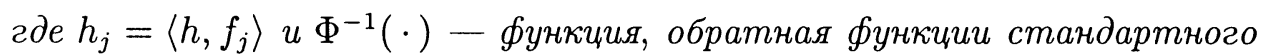
гауссовского распределения $\Phi(\cdot)$.

3 а м е ч а н и е. Рассматривая равенство (3) как усиленный закон больших чисел, нужно отметить, что речь идет о законе больших чисел для схемы серий, причем в пределах каждой серии случайные величины зависимы.

В том случае, когда о.н.б. $\left\{f_{k}\right\}$ совпадает с о.н.б. $\left\{e_{k}\right\}$ собственных векторов оператора $B$, утверждение теоремы 1 ранее было доказано автором в работе [1].

Следующая теорема дает более понятное, с вероятностной точки зрения, достаточное условие выполнения свойства (2).

Теорема 2. Если базис $\left\{f_{k}\right\} \subset \mathbf{H}$ является ортонормированным базисом собственных векторов ковариачионного оператора $\widetilde{B}$ гауссовской меры $\mu_{2}\{\cdot ; \widetilde{B}\}$, эквивалентной гауссовской мере $\mu_{2}\{\cdot ; B\}$, то имеет место сходимость ряда (2).

Не следует думать, что для каждой меры $\mu_{\alpha}\{\cdot ; B\}$ и для каждого о.н.б. $\left\{f_{k}\right\} \subset \mathbf{H}$ выполняется условие (2). В п. 5 настоящей работы приводится пример ковариационного оператора $B_{0}: \mathbf{H} \rightarrow \mathbf{H}$ и о.н.б. $\left\{f_{k}\right\} \subset \mathbf{H}$, для которых не выполняется соотношение (2), а сходимость (3) не имеет места, по крайней мере, для $\alpha=2$. 
Следует отметить, что близкие задачи выбора базиса возникают в теории вариационных методов приближенного решения операторных уравнений [2]-[4]. В этой теории при описании линейных операторов, допускающих редукцию относительно любого ортонормированного базиса гильбертова пространства, рассматривают правильные операторы. Предлагаемое доказательство теоремы 2 основано на том факте, что известное условие эквивалентности гауссовских мер, выраженное в терминах ковариационных операторов [5, гл. III, § 17, теорема 4], является частным случаем представления правильного оператора.

2. Обозначения. В добавление к семейству ортопроекторов $\left\{\pi_{m}\right\}$, определенных соотношением (1), вновь используя о.н.б. $\left\{f_{k}\right\} \subset \mathbf{H}$, рассмотрим семейство ортопроекторов $\left\{\pi_{m i}\right\}$ :

$$
\begin{gathered}
\text { если } h \in \mathbf{H} \quad \text { и } \quad h=\sum_{k=1}^{\infty}\left\langle h, f_{k}\right\rangle f_{k}, \\
\text { то } \pi_{m i} h=\sum_{k=1, k \neq i}^{m}\left\langle h, f_{k}\right\rangle f_{k}, \quad m=1,2, \ldots, \quad 1 \leqslant i \leqslant m .
\end{gathered}
$$

Заметим, что у оператора $\left(\pi_{m} B \pi_{m}\right): \mathbf{H}_{m} \rightarrow \mathbf{H}_{m}$ (ввиду того, что $\operatorname{ker} B=\{\boldsymbol{0}\})$ существует обратный. Матрицу этого оператора в базисе $\left\{f_{k}\right\}_{k=1, \ldots, m}$ обозначим следующим образом:

$$
B_{m}^{-1}=\left[\left\langle\left(\pi_{m} B \pi_{m}\right)^{-1} f_{i}, f_{j}\right\rangle\right]=\left[b_{(m)}^{i j}\right], \quad i, j=1, \ldots, m .
$$

Введем обозначения для следующих квадратичных форм:

$$
\begin{aligned}
s_{n}^{2}(h) & :=\frac{1}{n}\left\langle\left(\pi_{n} B \pi_{n}\right)^{-1} \pi_{n} h, \pi_{n} h\right\rangle, \\
s_{n, i}^{2}(h) & :=\frac{1}{n-1}\left\langle\left(\pi_{n i} B \pi_{n i}\right)^{-1} \pi_{n i} h, \pi_{n i} h\right\rangle, \\
q_{n}^{2}(h) & :=\frac{1}{n}\left\langle\left(\pi_{n} B \pi_{n}\right)^{-1}\left[\operatorname{diag}\left(\pi_{n} B \pi_{n}\right)^{-1}\right]^{-1}\left(\pi_{n} B \pi_{n}\right)^{-1} \pi_{n} h, \pi_{n} h\right\rangle,
\end{aligned}
$$

где $\operatorname{diag}\left(\pi_{n} B \pi_{n}\right)^{-1}$ - конечномерный линейный оператор с диагональной в базисе $\left(f_{1}, \ldots, f_{n}\right)$ матрицей $\operatorname{diag}\left\{b_{(n)}^{11}, b_{(n)}^{22}, \ldots, b_{(n)}^{n n}\right\}$.

Приведем несколько удобных формул для квадратичной формы $q_{n}^{2}(h)$ :

$$
q_{n}^{2}(h)=\frac{1}{n} \sum_{i=1}^{n} \frac{1}{b_{(n)}^{i i}}\left(\sum_{k=1}^{n} b_{(n)}^{i k} h_{k}\right)^{2}=\frac{1}{n} \sum_{i=1}^{n} \frac{\left\langle\left(\pi_{n} B \pi_{n}\right)^{-1} f_{i}, h\right\rangle^{2}}{\left\langle\left(\pi_{n} B \pi_{n}\right)^{-1} f_{i}, f_{i}\right\rangle} .
$$

Далее, учитывая формулу условной гауссовской плотности (теорема о нормальной корреляции $[6$, гл. 24, п. 24.6], [7, гл. II, §13, теорема 2]), 
будем иметь

$$
\begin{aligned}
\frac{1}{b_{(n)}^{i i}}\left(\sum_{k=1}^{n} b_{(n)}^{i k} h_{k}\right)^{2} & =\left\langle\left(\pi_{n} B \pi_{n}\right)^{-1} \pi_{n} h, \pi_{n} h\right\rangle-\left\langle\left(\pi_{n i} B \pi_{n i}\right)^{-1} \pi_{n i} h, \pi_{n i} h\right\rangle \\
& =\frac{\left[h_{i}-m_{i}\left(h^{(n)}(\hat{\imath})\right)\right]^{2}}{\sigma_{n}^{2}(\hat{\imath})}
\end{aligned}
$$

где $m_{i}\left(h^{(n)}(\hat{\imath})\right), \sigma_{n}^{2}(\hat{\imath})$ - условное среднее и условная дисперсия.

Таким образом,

$$
q_{n}^{2}(h)=\frac{1}{n} \sum_{i=1}^{n}\left[n s_{n}^{2}(h)-(n-1) s_{n, i}^{2}(h)\right] .
$$

Примем обозначения для множества сходимости и предельной случайной величины последовательности квадратичных форм $\left\{s_{n}^{2}(h)\right\}$ :

$$
s_{\infty}^{2}(h):=\lim _{n \rightarrow \infty} s_{n}^{2}(h), \quad \Gamma:=\left\{h \in \mathbf{H}: 0<s_{\infty}^{2}(h)<+\infty\right\} .
$$

Будем обозначать через $\mathbf{E}_{2}\{\cdot\}$ математическое ожидание по гауссовской мере

$$
\mu_{2}\{\cdot ; B\}: \mathbf{E}_{2}\{Z(h)\}:=\int_{\mathbf{H}} Z(h) \mu_{2}\{d h ; B\} .
$$

3. Вспомогательные утверждения. Сделаем несколько кратких замечаний о структуре этого пункта. В лемме 1 мы приводим явный вид представления Шенберга функций $F_{1 \ldots n}^{(\alpha)}$, с помощью которого дается представление условных распределений $F_{i \mid 1 \ldots \hat{\imath} \ldots n}^{(\alpha)}$ в виде непрерывной смеси гауссовских. Это представление позволяет устанавливать справедливость утверждений, относящихся к эллиптически контурированным устойчивым мерам, на основе соответствующих утверждений для гауссовских мер. В лемме 2 доказана $\delta$-образность вспомогательной последовательности $h_{n-1}^{(\alpha)}$. Этот факт является основой для «принципа локализации», используемого при доказательстве леммы 3.

Лемма 1 (представление Шенберга). Справедливь следующие равенства:

$$
\begin{aligned}
& \text { 1. } \exp \left\{-\langle B y, y\rangle^{\alpha / 2}\right\} \\
& =\int_{0}^{\infty} \exp \left\{-\frac{1}{2}\left\langle u^{2} B y, y\right\rangle\right\} g\left(\frac{u^{2}}{2} ; \frac{\alpha}{2}, 1\right) u d u \\
& 2^{\circ} . F_{1 \ldots n}^{(\alpha)}\left(x_{1}, \ldots, x_{n}\right)=\int_{0}^{\infty} \mu_{2}\left\{\left\langle h, f_{1}\right\rangle \leqslant x_{1}, \ldots,\left\langle h, f_{n}\right\rangle \leqslant x_{n} ; u^{2} B\right\} \\
& \times g\left(\frac{u^{2}}{2} ; \frac{\alpha}{2}, 1\right) u d u
\end{aligned}
$$


где $g(\cdot ; a, 1)$ - плотность одномерного крайнего устойчивого распределения с показателем $a \in] 0,1[($ см. [8, гл. 2, п. 2.5]);

$$
\begin{aligned}
3^{\circ} . & F_{i \mid 1 \ldots \hat{i} \ldots n}^{(\alpha)}\left(h_{i} \mid h_{1}, \ldots, \widehat{h}_{i}, \ldots, h_{n}\right) \\
= & \int_{0}^{\infty} \Phi\left(\left[\frac{n s_{n}^{2}(h)-(n-1) s_{n, i}^{2}(h)}{z}\right]^{1 / 2}\right) h_{n-1}^{(\alpha)}\left(s_{n, i}^{2}(h), z\right) d z
\end{aligned}
$$

$2 \partial e$

$$
h_{m}^{(\alpha)}(t, z)=\frac{\exp [-(m / 2)(t / z+\ln z)] g(z / 2 ; \alpha / 2,1)}{\int_{0}^{\infty} \exp [-(m / 2)(t / u+\ln u)] g(u / 2 ; \alpha / 2,1) d u} .
$$

Д о к а з а т е л ь с т в о. 1․ Формула (9) сразу следует из известного соотношения (см. [8, гл. 2, п. 2.10], [9, т. 2, гл. XIII, §6])

$$
\left.\exp \left(-\rho^{\alpha}\right)=\int_{0}^{\infty} \exp \left(-\rho^{2} v\right) g\left(v ; \frac{\alpha}{2}, 1\right) d v, \quad \alpha \in\right] 0,2[.
$$

$2^{\circ}$. Используя известную формулу для характеристического функционала гауссовской меры, можно утверждать, что выражения (9) и (10) связаны преобразованием Фурье-Стилтьеса. Поэтому справедливость этого пункта следует из теоремы единственности для характеристических функций.

$3^{\circ}$. Обозначим через $f_{1 \ldots n}^{(\alpha)}\left(x_{1}, \ldots, x_{n}\right)$ плотность распределения $F_{1 \ldots n}^{(\alpha)}\left(x_{1}, \ldots, x_{n}\right)$. Тогда из формулы $(9)$ получаем

$$
\begin{aligned}
& f_{1 \ldots n}^{(\alpha)}\left(x_{1}, \ldots, x_{n}\right)=\frac{1}{(2 \pi)^{n / 2}\left(\operatorname{det} B_{n}\right)^{1 / 2}} \\
& \quad \times \int_{0}^{\infty} \frac{1}{u^{n}} \exp \left\{-\frac{1}{2 u^{2}}\left\langle\left(\pi_{n} B \pi_{n}\right)^{-1} \pi_{n} x, \pi_{n} x\right\rangle\right\} g\left(\frac{u^{2}}{2} ; \frac{\alpha}{2}, 1\right) u d u .
\end{aligned}
$$

Отсюда, записывая выражение для условных плотностей в виде отношения безусловных и применяя теорему о нормальной корреляции, с учетом обозначений (4)-(6) будем иметь:

$$
\begin{aligned}
f_{i \mid 1 \ldots \hat{\imath} \ldots n}^{(\alpha)}\left(x_{i} \mid x_{1}, \ldots, \widehat{x}_{i}, \ldots, x_{n}\right) \\
=\int_{0}^{\infty} \frac{1}{\sqrt{2 \pi} \sigma_{n}(\hat{\imath}) u} \exp \left\{-\frac{\left[x_{i}-m_{i}\left(x^{(n)}(\hat{\imath})\right)\right]^{2}}{2 \sigma_{n}^{2}(\hat{\imath}) u^{2}}\right\} \\
\quad \times \frac{\exp \left\{-(n-1)\left[s_{n, i}^{2}(x) / u^{2}+\ln u^{2}\right] / 2\right\} g\left(u^{2} / 2 ; \alpha / 2,1\right) u}{\int_{0}^{\infty} \exp \left\{-(n-1)\left[s_{n, i}^{2}(x) / v^{2}+\ln v^{2}\right] / 2\right\} g\left(v^{2} / 2 ; \alpha / 2,1\right) v d v} d u .
\end{aligned}
$$

Таким образом, используя обозначение (12), получаем

$$
\begin{aligned}
& F_{i \mid 1 \ldots \hat{\imath} \ldots n}^{(\alpha)}\left(h_{i} \mid h_{1}, \ldots, \widehat{h}_{i}, \ldots, h_{n}\right) \\
& \quad=\int_{0}^{\infty} \Phi\left(\left[\frac{n s_{n}^{2}(h)-(n-1) s_{n, i}^{2}(h)}{z}\right]^{1 / 2}\right) h_{n-1}^{(\alpha)}\left(s_{n, i}^{2}(h), z\right) d z .
\end{aligned}
$$

Лемма 1 доказана. 
Лемма 2. Если $\lim _{n \rightarrow \infty} x_{n}=x_{\infty}>0$, mо последовательность $\left\{h_{n-1}^{(\alpha)}\left(x_{n}, z\right)\right\}$ является $\delta$-образной:

1. $h_{n-1}^{(\alpha)}\left(x_{n}, z\right)>0$ nрu $z>0$;

$2^{\circ}$. $\lim _{n \rightarrow \infty} h_{n-1}^{(\alpha)}\left(x_{n}, x_{n}\right)=+\infty$;

3. $\int_{0}^{\infty} h_{n-1}^{(\alpha)}\left(x_{n}, z\right) d z \equiv 1$;

4. $\lim _{n \rightarrow \infty} \int_{\tau_{n}^{-}}^{\tau_{n}^{+}} h_{n-1}^{(\alpha)}\left(x_{n}, z\right) d z=1$, zдe $\tau_{n}^{ \pm}=x_{n} \pm \sqrt{2} x_{n} n^{-1 / 4}$;

$5^{\circ}$. $\int_{\tau_{n}^{+}}^{\infty} h_{n-1}^{(\alpha)}\left(x_{n}, z\right) d z=O\left((n-1)^{1 / 2} \exp \left[-(n-1)^{1 / 2} / 4\right]\right)$.

Д ок азатель с т в о. Соотношения $1^{\circ}$ и $3^{\circ}$ очевидны. Что касается пп. $2^{\circ}, 4^{\circ}, 5^{\circ}$, то их справедливость устанавливается с помощью метода Лапласа нахождения асимптотики интегралов (см. [10, гл. II, $\S 2$, п. 2, теорема 2.2]). При этом доказывается, что $\left[\tau_{n}^{-}, \tau_{n}^{+}\right]$- отрезок влияния точки максимума $z=x_{n}$ функции $h_{n-1}^{(\alpha)}\left(x_{n}, z\right)$. Подробное доказательство этой леммы приведено в работе [1].

Рассмотрим последовательность вспомогательных функций

$$
A_{n}(u ; x)=\frac{1}{u} \Phi^{-1}\left(\int_{0}^{\infty} \Phi\left(\frac{u}{\sqrt{z}}\right) h_{n-1}^{(\alpha)}(x, z) d z\right),
$$

считая $u>0, x \geqslant 0, n=1,2, \ldots$, а функцию $h_{n-1}^{(\alpha)}(\cdot, \cdot)$ определенной соотношением (12).

Лемма 3. $1^{\circ}$. Если у последовательности неотрицательных иисел $\left\{x_{n}\right\}$ существует $\lim _{n \rightarrow \infty} x_{n}=x_{\infty}>0$, то для любой последовательности положительных чисел $\left\{u_{n}\right\}$ выполняется неравенство

$$
\varlimsup_{n \rightarrow \infty} A_{n}\left(u_{n} ; x_{n}\right) \leqslant \frac{1}{x_{\infty}^{1 / 2}} .
$$

$2^{\circ}$. Если у последовательности неотричательных чисел $\left\{x_{n}\right\}$ существует $\lim _{n \rightarrow \infty} x_{n}=x_{\infty}>0$, а для последовательности $\left\{u_{n}\right\}$ выполняется неравенство $0<u_{n}<n^{1 / k}\left(k=\mathrm{const} \geqslant 5, \forall n>N_{k}\right)$, то

$$
\lim _{n \rightarrow \infty} A_{n}\left(u_{n} ; x_{n}\right)=\frac{1}{x_{\infty}^{1 / 2}} \text {. }
$$

Д о к а з а т е л ь с т в а утверждений этой леммы приведены в работе [1] (леммы 10 и 11). Дадим краткое изложение этих доказательств. Прежде всего следует отметить, что неравенство (14) является следствием неравенства Иенсена

$$
A_{n}\left(u_{n} ; x_{n}\right) \leqslant \int_{0}^{\infty} \frac{1}{\sqrt{z}} h_{n-1}^{(\alpha)}\left(x_{n}, z\right) d z
$$

и «фильтрующего» свойства $\delta$-образной последовательности:

$$
\lim _{n \rightarrow \infty} \int_{0}^{\infty} \frac{1}{\sqrt{z}} h_{n-1}^{(\alpha)}\left(x_{n}, z\right) d z=\frac{1}{x_{\infty}^{1 / 2}} .
$$


Ограничимся изложением доказательства равенства (15) в случае, когда $\lim _{m \rightarrow \infty} u_{n_{m}}=+\infty$, поскольку только в этом случае используется ограничение на рост последовательности $\left\{u_{n}\right\}$.

Учитывая утверждение п. $1^{\circ}$, для доказательства соотношения (15) достаточно установить неравенство

$$
\varliminf_{n \rightarrow \infty} A_{n}\left(u_{n} ; x_{n}\right) \geqslant \frac{1}{x_{\infty}^{1 / 2}} .
$$

Для этого вначале, используя теорему о среднем, покажем, что

$$
A_{n}\left(u_{n} ; x_{n}\right) \geqslant \frac{1}{u_{n}} \Phi^{-1}\left(1-\left[1-\Phi\left(\frac{u_{n}}{\sqrt{\tau_{n}^{*}}}\right)\right]-\int_{\tau_{n}^{+}}^{\infty} h_{n-1}^{(\alpha)}\left(x_{n}, z\right) d z\right),
$$

где $\tau_{n}^{*} \in\left[\tau_{n}^{-}, \tau_{n}^{+}\right]$.

Далее, применяя известную асимптотическую формулу (см. [9, т. 1 , гл. VII, §1, лемма 2])

$$
1-\Phi\left(\frac{u_{n}}{\sqrt{\tau_{n}^{*}}}\right) \sim \frac{\sqrt{\tau_{n}^{*}}}{u_{n}} \frac{1}{\sqrt{2 \pi}} \exp \left(-\frac{u_{n}^{2}}{2 \tau_{n}^{*}}\right) \quad(n \rightarrow+\infty)
$$

и п. $5^{\circ}$ леммы 2 , с учетом ограничений $0<u_{n}<n^{1 / k}, k \geqslant 5$, получаем равенство

$$
\frac{\int_{\tau_{n}^{+}}^{\infty} h_{n-1}^{(\alpha)}\left(x_{n}, z\right) d z}{1-\Phi\left(u_{n} / \sqrt{\tau_{n}^{*}}\right)}=O\left(n^{(2+k) / k} \exp \left[-\frac{(n-1)^{1 / 2}}{8}\right]\right) .
$$

Используя это соотношение, неравенство (16) можно переписать в виде

$$
A_{n}\left(u_{n} ; x_{n}\right) \geqslant \frac{1}{u_{n}} \Phi^{-1}\left(1-\left[1-\Phi\left(\frac{u_{n}}{\sqrt{\tau_{n}^{*}}}\right)\right][1+o(1)]\right) .
$$

Обозначим

$$
\eta_{n}=\left[1-\Phi\left(\frac{u_{n}}{\sqrt{\tau_{n}^{*}}}\right)\right][1+o(1)],
$$

тогда неравенство (17) принимает вид

$$
A_{n}\left(u_{n} ; x_{n}\right) \geqslant \frac{1}{\sqrt{\tau_{n}^{*}}} \frac{\Phi^{-1}\left(1-\eta_{n}\right)}{\Phi^{-1}\left(1-\eta_{n} /(1+o(1))\right)} .
$$

Но так как $\eta_{n} \rightarrow 0$ и $\tau_{n}^{*} \rightarrow x_{\infty}$ при $n \rightarrow+\infty$, а

$$
\lim _{n \rightarrow \infty} \frac{\Phi^{-1}\left(1-\eta_{n}\right)}{\Phi^{-1}\left(1-\eta_{n} /(1+o(1))\right)}=1,
$$

то $\underline{\lim }_{n \rightarrow \infty} A_{n}\left(u_{n} ; x_{n}\right) \geqslant 1 / x_{\infty}^{1 / 2}$. Лемма 3 доказана. 
Лемма 4. Последовательности $s_{n}^{2}(h) u s_{n, i(n)}^{2}(h)$ обладают относительно гауссовской меры $\mu_{2}\{\cdot ; B\}$ следуюшими свойствами.

$1^{\circ}$. Для почти всех $h \in \mathbf{H}$

$$
\lim _{n \rightarrow \infty} s_{n}^{2}(h)=1 .
$$

$2^{\circ}$. Для любой последовательности натуральных чисел $i(n)$ $(1 \leqslant i(n) \leqslant n)$ u для почти всех $h \in \mathbf{H}$

$$
\lim _{n \rightarrow \infty} s_{n, i(n)}^{2}(h)=1 .
$$

Д о к а з а т е л ь с т в о. Вначале приведем квадратичные формы $s_{n}^{2}(h)$ к сумме квадратов. Для этого введем вспомогательную последовательность линейных функционалов

$$
\xi_{i}^{(n)}(h):=\left\langle\left(\pi_{n} B \pi_{n}\right)^{-1 / 2} f_{i}, h\right\rangle, \quad i=1, \ldots, n .
$$

Относительно гауссовской меры $\mu_{2}\{\cdot ; B\}$ эти функционалы образуют последовательность независимых $(0,1)$-гауссовских случайных величин. Далее, так как

$$
\left(\pi_{n} B \pi_{n}\right)^{-1 / 2} \pi_{n} h=\sum_{i=1}^{n} \xi_{i}^{(n)}(h) f_{i}
$$

To

$$
\left\langle\left(\pi_{n} B \pi_{n}\right)^{-1} \pi_{n} h, \pi_{n} h\right\rangle=\left\langle\left(\pi_{n} B \pi_{n}\right)^{-1 / 2} \pi_{n} h,\left(\pi_{n} B \pi_{n}\right)^{-1 / 2} \pi_{n} h\right\rangle=\sum_{i=1}^{n} \xi_{i}^{(n)}(h)^{2} .
$$

Следовательно,

$$
s_{n}^{2}(h)=\frac{1}{n} \sum_{i=1}^{n} \xi_{i}^{(n)}(h)^{2} .
$$

Для доказательства п. $1^{\circ}$ осталось воспользоваться усиленным законом больших чисел в форме Кантелли [7, гл. IV , § 3, теорема 1]. В самом деле, используя признак сходимости почти наверное и неравенство Чебышева, будем иметь

$$
\begin{aligned}
& \sum_{n=1}^{\infty} \mu_{2}\left\{\frac{1}{n}\left|\sum_{i=1}^{n}\left[\xi_{i}^{(n)}(h)^{2}-1\right]\right|>\varepsilon ; B\right\} \\
& \leqslant \frac{1}{\varepsilon^{4}} \sum_{n=1}^{\infty} \frac{1}{n^{4}} \mathbf{E}_{2}\left\{\left(\sum_{i=1}^{n}\left[\xi_{i}^{(n)}(h)^{2}-1\right]\right)^{4}\right\} \\
& =\frac{1}{\varepsilon^{4}} \sum_{n=1}^{\infty} \frac{1}{n^{4}}\left[\sum_{i=1}^{n} \mathbf{E}_{2}\left\{\left(\xi_{i}^{(n)}(h)^{2}-1\right)^{4}\right\}\right. \\
& \left.\quad+6 \sum_{i, j=1, i<j}^{n} \mathbf{E}_{2}\left\{\left[\xi_{i}^{(n)}(h)^{2}-1\right]^{2}\right\} \mathbf{E}_{2}\left\{\left[\xi_{j}^{(n)}(h)^{2}-1\right]^{2}\right\}\right] \\
& =\frac{1}{\varepsilon^{4}} \sum_{n=1}^{\infty} \frac{1}{n^{4}}[60 n+12 n(n-1)]<+\infty .
\end{aligned}
$$


2. Как было показано выше (см. формулы (7) и (8)),

$$
s_{n}^{2}(h)-\frac{n-1}{n} s_{n, i(n)}^{2}(h)=\frac{1}{n} \frac{\left\langle\left(\pi_{n} B \pi_{n}\right)^{-1} f_{i(n)}, h\right\rangle^{2}}{\left\langle\left(\pi_{n} B \pi_{n}\right)^{-1} f_{i(n)}, f_{i(n)}\right\rangle} .
$$

Нетрудно показать, что относительно гауссовской меры $\mu_{2}\{\cdot ; B\}$ случайные величины

$$
\frac{\left\langle\left(\pi_{n} B \pi_{n}\right)^{-1} f_{i(n)}, h\right\rangle}{\left\langle\left(\pi_{n} B \pi_{n}\right)^{-1} f_{i(n)}, f_{i(n)}\right\rangle^{1 / 2}}, \quad n=1,2, \ldots,
$$

являются $(0,1)$-гауссовскими. Поэтому, используя неравенство Чебышева, для любого $\varepsilon>0$ получаем

$$
\begin{aligned}
& \sum_{n=1}^{\infty} \mu_{2}\left\{s_{n}^{2}(h)-\frac{n-1}{n} s_{n, i(n)}^{2}(h)>\varepsilon ; B\right\} \\
& \quad \leqslant \frac{1}{\varepsilon^{2}} \sum_{n=1}^{\infty} \frac{1}{n^{2}} \mathbf{E}_{2}\left\{\frac{\left\langle\left(\pi_{n} B \pi_{n}\right)^{-1} f_{i(n)}, h\right\rangle^{4}}{\left\langle\left(\pi_{n} B \pi_{n}\right)^{-1} f_{i(n)}, f_{i(n)}\right\rangle^{2}}\right\}=\frac{1}{\varepsilon^{2}} \sum_{n=1}^{\infty} \frac{3}{n^{2}}<+\infty
\end{aligned}
$$

Следовательно, для $\mu_{2}\{\cdot ; B\}$-почти всех $h \in \mathbf{H}$

$$
\lim _{n \rightarrow \infty}\left[s_{n}^{2}(h)-\frac{n-1}{n} s_{n, i(n)}^{2}(h)\right]=0
$$

и, таким образом, п. $2^{\circ}$ следует из п. $1^{\circ}$. Лемма доказана.

3 а м е ч а н и е. Можно показать, что последовательность случайных величин $\left\{n s_{n}^{2}(h)-n\right\}, h \in \mathbf{H}, n=1,2, \ldots$, образует квадратично интегрируемый мартингал с квадратической характеристикой, равной $2 n$.

Лемма 5. Для того итобы последовательность квадратичных борм $\left\{q_{n}^{2}(h)\right\}$ сходилась по гауссовской мере $\mu_{2}\{\cdot ; B\} \kappa$ единиче, необходимо и достаточно, чтобы выполнялось условие

$$
\lim _{n \rightarrow \infty} \frac{1}{n^{2}} \sum_{i=1}^{n} \sum_{j=1}^{n} \frac{\left\langle\left(\pi_{n} B \pi_{n}\right)^{-1} f_{i}, f_{j}\right\rangle^{2}}{\left\langle\left(\pi_{n} B \pi_{n}\right)^{-1} f_{i}, f_{i}\right\rangle\left\langle\left(\pi_{n} B \pi_{n}\right)^{-1} f_{j}, f_{j}\right\rangle}=0 .
$$

Д о к а з а т е л с т в о. Достаточность условия (19) следует из неравенства Чебышева, так как по известным формулам для интегралов по гауссовским мерам (см. [11, гл. II, §2, п. 3])

$$
\begin{aligned}
\mathbf{E}_{2}\left\{q_{n}^{2}(h)\right\} & =\frac{1}{n} \operatorname{tr}\left(\pi_{n} B \pi_{n}\right)^{-1}\left[\operatorname{diag}\left(\pi_{n} B \pi_{n}\right)^{-1}\right]^{-1}=1 \\
\mathbf{E}_{2}\left\{\left[q_{n}^{2}(h)-1\right]^{2}\right\} & =\mathbf{E}_{2}\left\{q_{n}^{4}(h)\right\}-1 \\
& =\frac{2}{n^{2}} \operatorname{tr}\left\{\left(\pi_{n} B \pi_{n}\right)^{-1}\left[\operatorname{diag}\left(\pi_{n} B \pi_{n}\right)^{-1}\right]^{-1}\right\}^{2} \\
& =\frac{2}{n^{2}} \sum_{i=1}^{n} \sum_{j=1}^{n} \frac{\left\langle\left(\pi_{n} B \pi_{n}\right)^{-1} f_{i}, f_{j}\right\rangle^{2}}{\left\langle\left(\pi_{n} B \pi_{n}\right)^{-1} f_{i}, f_{i}\right\rangle\left\langle\left(\pi_{n} B \pi_{n}\right)^{-1} f_{j}, f_{j}\right\rangle} .
\end{aligned}
$$


Докажем необходимость условия (19). С одной стороны, справедливость этого условия можно установить, вычисляя $\psi_{n}(t)$ - характеристические функции квадратичных форм $q_{n}^{2}(h)$ и рассматривая сходимость

$$
\left.\lim _{n \rightarrow \infty} \psi_{n}(t)=e^{i t}, \quad \forall t \in\right]-\infty,+\infty[.
$$

С другой стороны, утверждение леммы 5 следует из соотношений (20) ввиду того известного факта, что последовательность многочленов второго (или даже большего) порядка сходится по гауссовской мере $\mu_{2}\{\cdot ; B\}$ тогда и только тогда, когда она сходится по норме в пространствах $\mathbf{L}^{p}\left(\mu_{2}\{\cdot ; B\}\right)$ для всех $p \in[1, \infty[$ (см. [12, п. 5.6.5]). Лемма доказана.

Лемма 6. Если сходится ряд (2), то для $\mu_{2}\{\cdot ; B\}$-почти всех $h \in \mathbf{H}$

$$
\lim _{n \rightarrow \infty} q_{n}^{2}(h)=1 .
$$

Д о к а з а т е л ь с в о. Запишем разложение квадратичной формы $n q_{n}^{2}(h)$ по системе (18) случайных величин $\left\{\xi_{i}^{(n)}(h)\right\}$ :

$$
\begin{aligned}
n q_{n}^{2}(h)= & \sum_{i=1}^{n} \sum_{j=1}^{n}\left\langle\left(\pi_{n} B \pi_{n}\right)^{-1 / 2}\left[\operatorname{diag}\left(\pi_{n} B \pi_{n}\right)^{-1}\right]^{-1}\left(\pi_{n} B \pi_{n}\right)^{-1 / 2} f_{i}, f_{j}\right\rangle \\
& \times \xi_{i}^{(n)}(h) \xi_{j}^{(n)}(h) .
\end{aligned}
$$

Введем сокращающее обозначение

$$
D_{n}=\left(\pi_{n} B \pi_{n}\right)^{-1 / 2}\left[\operatorname{diag}\left(\pi_{n} B \pi_{n}\right)^{-1}\right]^{-1}\left(\pi_{n} B \pi_{n}\right)^{-1 / 2}
$$

и воспользуемся (см. $[13$, гл. $4, \S \S 1,3])$ представлением квадратичной формы $n q_{n}^{2}(h)$ в виде суммы мартингал-разностей:

$$
n q_{n}^{2}(h)-n=\sum_{k=1}^{n} \gamma_{k n}(h)
$$

где

$$
\gamma_{k n}(h)=\left\langle D_{n} f_{k}, f_{k}\right\rangle\left\{\left[\xi_{k}^{(n)}(h)\right]^{2}-1\right\}+2 \xi_{k}^{(n)}(h) \sum_{j=k+1}^{n}\left\langle D_{n} f_{k}, f_{j}\right\rangle \xi_{j}^{(n)}(h) .
$$

Вычислим моменты величин $\gamma_{k n}(h): \mathbf{E}_{2}\left\{\gamma_{k n}(h)\right\} \equiv 0$;

$$
\begin{aligned}
\mathbf{E}_{2}\left\{\gamma_{k n}^{2}(h)\right\}=2\left\langle D_{n} f_{k}, f_{k}\right\rangle^{2}+4 \sum_{j=k+1}^{n}\left\langle D_{n} f_{k}, f_{j}\right\rangle^{2} \\
\mathbf{E}_{2}\left\{\gamma_{i n}(h) \gamma_{j n}(h)\right\}=0, \quad i \neq j ; \\
\mathbf{E}_{2}\left\{\gamma_{k n}^{4}(h)\right\}=60\left\langle D_{n} f_{k}, f_{k}\right\rangle^{4}+240\left\langle D_{n} f_{k}, f_{k}\right\rangle^{2} \sum_{j=k+1}^{n}\left\langle D_{n} f_{k}, f_{j}\right\rangle^{2} \\
\quad+144\left(\sum_{j=k+1}^{n}\left\langle D_{n} f_{k}, f_{j}\right\rangle^{2}\right)^{2}
\end{aligned}
$$


Теперь воспользуемся усиленным законом больших чисел в форме Кантелли для сумм мартингал-разностей (см. $[13$, гл. $4, \S 10]):$ если для каждого натурального $n$ случайнье величины $\left\{\xi_{i}^{(n)}(h)\right\}_{i=1, \ldots, n}$ независимы $u$

$$
\sum_{n=1}^{\infty}\left(\frac{1}{n^{2}} \sum_{k=1}^{n} \sqrt{\mathbf{E}_{2}\left\{\gamma_{k n}^{4}(h)\right\}}\right)^{2}<+\infty
$$

то почти наверное по мере $\mu_{2}\{\cdot ; B\}$

$$
\lim _{n \rightarrow \infty} q_{n}^{2}(h)=1 \text {. }
$$

Покажем, что из условия (2) следует (24). В самом деле, из соотношений (21) и (23) получаем неравенство

$$
\mathbf{E}_{2}\left\{\gamma_{k n}^{4}(h)\right\} \leqslant 60\left[\left\langle D_{n} f_{k}, f_{k}\right\rangle^{2}+2 \sum_{j=k+1}^{n}\left\langle D_{n} f_{k}, f_{j}\right\rangle^{2}\right]^{2}=15 \mathbf{E}_{2}\left\{\gamma_{k n}^{2}(h)\right\}^{2} .
$$

Отсюда, учитывая $(20)$ и $(22)$, будем иметь

$$
\begin{aligned}
\sum_{k=1}^{n} \sqrt{\mathbf{E}_{2}\left\{\gamma_{k n}^{4}(h)\right\}} & \leqslant \sqrt{15} \sum_{k=1}^{n} \mathbf{E}_{2}\left\{\gamma_{k n}^{2}(h)\right\}=\sqrt{15} \mathbf{E}_{2}\left\{\left[n q_{n}^{2}(h)-n\right]^{2}\right\} \\
& =2 \sqrt{15} \sum_{i=1}^{n} \sum_{j=1}^{n} \frac{\left\langle\left(\pi_{n} B \pi_{n}\right)^{-1} f_{i}, f_{j}\right\rangle^{2}}{\left\langle\left(\pi_{n} B \pi_{n}\right)^{-1} f_{i}, f_{i}\right\rangle\left\langle\left(\pi_{n} B \pi_{n}\right)^{-1} f_{j}, f_{j}\right\rangle}
\end{aligned}
$$

Следовательно,

$$
\begin{aligned}
& \sum_{n=1}^{\infty}\left(\frac{1}{n^{2}} \sum_{k=1}^{n} \sqrt{\mathbf{E}_{2}\left\{\gamma_{k n}^{4}(h)\right\}}\right)^{2} \\
& \quad \leqslant 60 \sum_{n=1}^{\infty}\left[\frac{1}{n^{2}} \sum_{i=1}^{n} \sum_{j=1}^{n} \frac{\left\langle\left(\pi_{n} B \pi_{n}\right)^{-1} f_{i}, f_{j}\right\rangle^{2}}{\left\langle\left(\pi_{n} B \pi_{n}\right)^{-1} f_{i}, f_{i}\right\rangle\left\langle\left(\pi_{n} B \pi_{n}\right)^{-1} f_{j}, f_{j}\right\rangle}\right]^{2} .
\end{aligned}
$$

Лемма доказана.

Лемма 7. Для любого $\alpha \in] 0,2[$ предельная случайная величина $s_{\infty}^{2}(h)$ обладает следующими свойствами.

$1^{\circ}$. Для $\mu_{\alpha}\{\cdot ; B\}$-почти всех $h \in \mathbf{H}$

$$
\lim _{n \rightarrow \infty} s_{n}^{2}(h)=s_{\infty}^{2}(h) .
$$

2․ Для любой последовательности натуральных чисел $i(n)$ $(1 \leqslant i(n) \leqslant n)$ u dля $\mu_{\alpha}\{\cdot ; B\}$-почти всех $h \in \mathbf{H}$

$$
\lim _{n \rightarrow \infty} s_{n, i(n)}^{2}(h)=s_{\infty}^{2}(h) .
$$


3․ При вьполнении условия (2) для $\mu_{\alpha}\{\cdot ; B\}$-почти всех $h \in \mathbf{H}$

$$
\lim _{n \rightarrow \infty} q_{n}^{2}(h)=s_{\infty}^{2}(h)
$$

$4^{\circ} . \mu_{\alpha}\left\{s_{\infty}^{2}(h) \leqslant u ; B\right\}=\int_{0}^{u / 2} g(t ; \alpha / 2,1) d t$.

Д ок азат ел ь с т в о. $1^{\circ}$. Выберем произвольное $\sigma>0$ и рассмотрим $\mu_{2}\left\{\cdot ; \sigma^{2} B\right\}$ - гауссовскую меру с нулевым средним и ковариационным оператором $\sigma^{2} B$. Из леммы 4 следует, что при $n \rightarrow+\infty$

$$
s_{n}^{2}(h) \rightarrow \sigma^{2} \quad \text { п.н. по мере } \mu_{2}\left\{\cdot ; \sigma^{2} B\right\} .
$$

Таким образом (см. [7, гл. II, §10, п. 3, теорема 1]), для любых $\sigma>0$ и $\varepsilon>0$

$$
\lim _{n \rightarrow \infty} \mu_{2}\left\{\sup _{k \geqslant 0}\left|s_{n+k}^{2}(h)-s_{n}^{2}(h)\right|>\varepsilon ; \sigma^{2} B\right\}=0 .
$$

Отсюда, используя представление Шенберга (10), получаем фундаментальность п.н. последовательности $\left\{s_{n}^{2}(h)\right\}$ по устойчивой мере $\mu_{\alpha}\{\cdot B\}$ :

$$
\lim _{n \rightarrow \infty} \mu_{\alpha}\left\{\sup _{k \geqslant 0}\left|s_{n+k}^{2}(h)-s_{n}^{2}(h)\right|>\varepsilon ; B\right\}=0 .
$$

Следовательно, можно утверждать (см. [7, гл. II, § 10, п. 5, теорема 4]), что существуют $\mathscr{B}(\mathbf{H})$-измеримая случайная величина $s_{\infty, \alpha}^{2}(h)$ и множество сходимости $\Gamma_{\alpha} \in \mathscr{B}(\mathbf{H})$ такие, что $\mu_{\alpha}\left\{\Gamma_{\alpha} ; B\right\}=1$ и

$$
\lim _{n \rightarrow \infty} s_{n}^{2}(h)=s_{\infty, \alpha}^{2}(h), \quad \forall h \in \Gamma_{\alpha} .
$$

С другой стороны, $\Gamma_{\alpha} \subseteq \Gamma, s_{\infty, \alpha}^{2}(h)=s_{\infty}^{2}(h), h \in \Gamma_{\alpha}$ и $\mu_{\alpha}\{\Gamma ; B\}=1$.

Можно считать, что $s_{\infty, \alpha}^{2}(h)>0$ для любого $h \in \Gamma_{\alpha}$, так как $\mu_{\alpha}\left\{s_{\infty, \alpha}^{2}(h)=0 ; B\right\}=0$ (см. п. $4^{\circ}$ этой леммы).

$2^{\circ}$. На основании п. $2^{\circ}$ леммы 4 можно утверждать, что для любого $\sigma>0$

$$
\lim _{n \rightarrow \infty}\left[s_{n}^{2}(h)-s_{n, i(n)}^{2}(h)\right]=0 \quad \text { п.н. по мере } \quad \mu_{2}\left\{\cdot ; \sigma^{2} B\right\}
$$

Повторяя рассуждения п. $1^{\circ}$, получаем

$$
\lim _{n \rightarrow \infty}\left[s_{n}^{2}(h)-s_{n, i(n)}^{2}(h)\right]=0 \quad \text { п.н. по мере } \quad \mu_{\alpha}\{\cdot ; B\} .
$$

Теперь доказательство п. $2^{\circ}$ следует из п. $1^{\circ}$ настоящей леммы.

$3^{\circ}$. На основании леммы 6 можно утверждать, что для любого $\sigma>0$

$$
\lim _{n \rightarrow \infty}\left[s_{n}^{2}(h)-q_{n}^{2}(h)\right]=0 \quad \text { п.н. по мере } \quad \mu_{2}\left\{\cdot ; \sigma^{2} B\right\} \text {. }
$$

Далее доказательство буквально повторяет рассуждения п. $2^{\circ}$. 
$4^{\circ}$. Как следует из (25), для любого $\sigma>0$

$$
\mu_{2}\left\{s_{\infty}^{2}(h) \leqslant u ; \sigma^{2} B\right\}=\left\{\begin{array}{lll}
0, & \text { когда } & u<\sigma^{2}, \\
1, & \text { когда } u>\sigma^{2} .
\end{array}\right.
$$

Поэтому, вновь пользуясь представлением Шенберга (10), получаем:

$$
\mu_{\alpha}\left\{s_{\infty}^{2}(h) \leqslant u ; B\right\}=\int_{0}^{\sqrt{u}} g\left(\frac{\sigma^{2}}{2} ; \frac{\alpha}{2}, 1\right) \sigma d \sigma=\int_{0}^{u / 2} g\left(t ; \frac{\alpha}{2}, 1\right) d t .
$$

Лемма доказана.

\section{Лемма 8. Для любого натурального $k$}

$$
\mu_{\alpha}\left\{\bigcup_{m=1}^{\infty} \bigcap_{n=m}^{\infty}\left\{h: \sup _{i=1, \ldots, n} \frac{\left\langle\left(\pi_{n} B \pi_{n}\right)^{-1} f_{i}, h\right\rangle^{2}}{\left\langle\left(\pi_{n} B \pi_{n}\right)^{-1} f_{i}, f_{i}\right\rangle} \leqslant n^{2 / k}\right\} ; B\right\}=1 .
$$

Д о к а з а т е л ь с т в о. Установим справедливость утверждения леммы вначале для гауссовской меры $\mu_{2}\left\{\cdot ; s^{2} B\right\}, s>0$.

Обозначим для краткости:

$$
W_{n}^{(k)}:=\left\{h: \sup _{i=1, \ldots, n} \frac{\left\langle\left(\pi_{n} B \pi_{n}\right)^{-1} f_{i}, h\right\rangle^{2}}{\left\langle\left(\pi_{n} B \pi_{n}\right)^{-1} f_{i}, f_{i}\right\rangle} \leqslant n^{2 / k}\right\}, \quad V_{k}:=\bigcup_{m=1}^{\infty} \bigcap_{n=m}^{\infty} W_{n}^{(k)} .
$$

Тогда из включений

$$
\bigcap_{n=m}^{\infty} W_{n}^{(k)} \subseteq \bigcap_{n=m+1}^{\infty} W_{n}^{(k)}, \quad m=1,2, \ldots,
$$

следует равенство

$$
\mu_{2}\left\{V_{k} ; s^{2} B\right\}=\lim _{m \rightarrow \infty} \mu_{2}\left\{\bigcap_{n=m}^{\infty} W_{n}^{(k)} ; s^{2} B\right\} .
$$

Далее, переходя к дополнениям, получаем

$$
\mu_{2}\left\{\bigcap_{n=m}^{\infty} W_{n}^{(k)} ; s^{2} B\right\} \geqslant 1-\sum_{n=m}^{\infty} \mu_{2}\left\{\overline{W_{n}^{(k)}} ; s^{2} B\right\} .
$$

Установим сходимость ряда в правой части (26). Так как

$$
\begin{aligned}
\mu_{2}\left\{\overline{W_{n}^{(k)}} ; s^{2} B\right\} & =\mu_{2}\left\{h: \sup _{i=1, \ldots, n} \frac{\left\langle\left(\pi_{n} B \pi_{n}\right)^{-1} f_{i}, h\right\rangle^{2}}{\left\langle\left(\pi_{n} B \pi_{n}\right)^{-1} f_{i}, f_{i}\right\rangle}>n^{2 / k} ; s^{2} B\right\} \\
& \leqslant \sum_{i=1}^{n} \mu_{2}\left\{h: \frac{\left\langle\left(\pi_{n} B \pi_{n}\right)^{-1} f_{i}, h\right\rangle^{2}}{\left\langle\left(\pi_{n} B \pi_{n}\right)^{-1} f_{i}, f_{i}\right\rangle}>n^{2 / k} ; s^{2} B\right\} \\
& =\sum_{i=1}^{n} 2\left[1-\Phi\left(\frac{n^{1 / k}}{s}\right)\right]=2 n\left[1-\Phi\left(\frac{n^{1 / k}}{s}\right)\right],
\end{aligned}
$$


то, пользуясь известным неравенством (см. $[9$, т. 1 , гл. VII, § 1, лемма 2])

$$
1-\Phi(u)<u^{-1} \frac{1}{\sqrt{2 \pi}} e^{-u^{2} / 2} \quad(u>0)
$$

будем иметь:

$$
\sum_{n=m}^{\infty} \mu_{2}\left\{\overline{W_{n}^{(k)}} ; s^{2} B\right\} \leqslant \sqrt{\frac{2}{\pi}} s \sum_{n=m}^{\infty} n^{1-1 / k} \exp \left\{-\frac{n^{2 / k}}{2 s^{2}}\right\}<+\infty
$$

для любого $s>0$. Теперь можно перейти к пределу в неравенстве (26). Тогда для любого $s>0$ получаем

$$
\mu_{2}\left\{V_{k} ; s^{2} B\right\} \geqslant 1-\lim _{m \rightarrow \infty} \sum_{n=m}^{\infty} \mu_{2}\left\{\overline{W_{n}^{(k)}} ; s^{2} B\right\}=1 .
$$

Для доказательства леммы осталось воспользоваться представлением Шенберга (10).

4. Доказательства теорем. Д ок а з а т ел ь с т о т е о ре м ы. Из леммы 7 следует сушествование множества $\widetilde{\Gamma} \in \mathscr{B}(\mathbf{H})$ (с мерой $\mu_{\alpha}\{\widetilde{\Gamma} ; B\} \equiv 1$ ), для каждого элемента которого выполняются пп. $1^{\circ}-3^{\circ}$ этой леммы. Кроме того, ввиду п. $4^{\circ}$ леммы 7 можно считать, что $s_{\infty}^{2}(h) \neq 0$ для любого $h \in \widetilde{\Gamma}$. Установим справедливость следующего неравенства: если $h \in \widetilde{\Gamma}$, то

$$
\varlimsup_{n \rightarrow \infty} \frac{1}{n} \sum_{i=1}^{n}\left[\Phi^{-1}\left(F_{i \mid 1 \ldots \ldots \ldots n}^{(\alpha)}\left(h_{i} \mid h_{1}, \ldots, \widehat{h_{i}}, \ldots, h_{n}\right)\right)\right]^{2} \leqslant 1 .
$$

Действительно, из формул (11), (12) следует соотношение

$$
\begin{aligned}
& \frac{1}{n} \sum_{i=1}^{n}\left[\Phi^{-1}\left(F_{i \mid 1 \ldots \hat{\imath} \ldots n}^{(\alpha)}\left(h_{i} \mid h_{1}, \ldots, \widehat{h}_{i}, \ldots, h_{n}\right)\right)\right]^{2} \\
& \leqslant \sup _{1 \leqslant i \leqslant n} A_{n}^{2}\left(\left[n s_{n}^{2}(h)-(n-1) s_{n, i}^{2}(h)\right]^{1 / 2} ; s_{n, i}^{2}(h)\right) \\
& \quad \times \frac{1}{n} \sum_{i=1}^{n}\left[n s_{n}^{2}(h)-(n-1) s_{n, i}^{2}(h)\right] \\
& =A_{n}^{2}\left(\left[n s_{n}^{2}(h)-(n-1) s_{n, i(n)}^{2}(h)\right]^{1 / 2} ; s_{n, i(n)}^{2}(h)\right) q_{n}^{2}(h),
\end{aligned}
$$

где $i(n)$ - натуральное число, на котором достигается супремум. Отсюда, используя п. $1^{\circ}$ леммы 3 и пп. $2^{\circ}, 3^{\circ}$ леммы 7 , получаем неравенство (27):

$$
\varlimsup_{n \rightarrow \infty} \frac{1}{n} \sum_{i=1}^{n}\left[\Phi^{-1}\left(F_{i \mid 1 \ldots \hat{z} \ldots n}^{(\alpha)}\left(h_{i} \mid h_{1}, \ldots, \widehat{h}_{i}, \ldots, h_{n}\right)\right)\right]^{2} \leqslant \frac{1}{s_{\infty}^{2}(h)} s_{\infty}^{2}(h)=1 .
$$


Покажем теперь, что при $h \in \widetilde{\Gamma} \cap V_{5}$ (см. обозначение множества $V_{k}$ в доказательстве леммы 8)

$$
\varliminf_{n \rightarrow \infty} \frac{1}{n} \sum_{i=1}^{n}\left[\Phi^{-1}\left(F_{i \mid 1 \ldots \hat{\imath} \ldots n}^{(\alpha)}\left(h_{i} \mid h_{1}, \ldots, \widehat{h_{i}}, \ldots, h_{n}\right)\right)\right]^{2} \geqslant 1 .
$$

В самом деле, элементы $h \in V_{5}$ (см. лемму 8) обладают следующим свойством: существует натуральное $m=m(h)$ такое, что

$$
\sup _{1 \leqslant i \leqslant n}\left|\frac{\left\langle\left(\pi_{n} B \pi_{n}\right)^{-1} f_{i}, h\right\rangle}{\left\langle\left(\pi_{n} B \pi_{n}\right)^{-1} f_{i}, f_{i}\right\rangle^{1 / 2}}\right| \leqslant n^{1 / 5}
$$

для всех $n \geqslant m(h)$. С другой стороны, из формул (7), (13) следует при $n \geqslant m(h)$ неравенство

$$
\begin{aligned}
\frac{1}{n} \sum_{i=1}^{n} & {\left[\Phi^{-1}\left(F_{i \mid 1 \ldots i \ldots n}^{(\alpha)}\left(h_{i} \mid h_{1}, \ldots, \widehat{h}_{i}, \ldots, h_{n}\right)\right)\right]^{2} } \\
\geqslant & \inf _{m(h) \leqslant i \leqslant n} A_{n}^{2}\left(\left|\frac{\left\langle\left(\pi_{n} B \pi_{n}\right)^{-1} f_{i}, h\right\rangle}{\left\langle\left(\pi_{n} B \pi_{n}\right)^{-1} f_{i}, f_{i}\right\rangle^{1 / 2}}\right| ; s_{n, i}^{2}(h)\right) \\
& \times \frac{1}{n} \sum_{i=m(h)}^{n} \frac{\left\langle\left(\pi_{n} B \pi_{n}\right)^{-1} f_{i}, h\right\rangle^{2}}{\left\langle\left(\pi_{n} B \pi_{n}\right)^{-1} f_{i}, f_{i}\right\rangle} \\
= & A_{n}^{2}\left(\left|\frac{\left\langle\left(\pi_{n} B \pi_{n}\right)^{-1} f_{j(n)}, h\right\rangle}{\left\langle\left(\pi_{n} B \pi_{n}\right)^{-1} f_{j(n)}, f_{j(n)}\right\rangle^{1 / 2}}\right| ; s_{n, j(n)}^{2}(h)\right) \\
& \times \frac{1}{n} \sum_{i=m(h)}^{n} \frac{\left\langle\left(\pi_{n} B \pi_{n}\right)^{-1} f_{i}, h\right\rangle^{2}}{\left\langle\left(\pi_{n} B \pi_{n}\right)^{-1} f_{i}, f_{i}\right\rangle},
\end{aligned}
$$

где $j(n)$ - натуральное число, на котором достигается инфимум. Осталось заметить, что свойство (29) позволяет использовать п. $2^{\circ}$ леммы 3 для перехода к пределу в неравенстве (30). Вновь используя пп. $2^{\circ}, 3^{\circ}$ леммы 7 , будем иметь для $h \in \widetilde{\Gamma} \cap V_{5}$ :

$$
\varliminf_{n \rightarrow \infty} \frac{1}{n} \sum_{i=1}^{n}\left[\Phi^{-1}\left(F_{i \mid 1 \ldots \hat{\imath} \ldots n}^{(\alpha)}\left(h_{i} \mid h_{1}, \ldots, \widehat{h_{i}}, \ldots, h_{n}\right)\right)\right]^{2} \geqslant \frac{1}{s_{\infty}^{2}(h)} s_{\infty}^{2}(h)=1 .
$$

Утверждение теоремы следует из неравенств (27) и (28), а также того факта (см. лемму 8), что $\mu_{\alpha}\{\widetilde{\Gamma} ; B\}=1$.

Д о к а за т е л ь с т в о т е о ре м ы 2. Вначале проведем оценку величины

$$
\left\langle\left(\pi_{n} B \pi_{n}\right)^{-1} f_{i}, f_{j}\right\rangle, \quad i, j=1, \ldots, n .
$$

Используя условие эквивалентности гауссовских мер в гильбертовом пространстве, можно утверждать (см. [5, гл. III, §17, теорема 4], [11, гл. II, §4, п. 3]), что $\operatorname{ker} B=\operatorname{ker} \widetilde{B}, B^{1 / 2} \mathbf{H}=\widetilde{B}^{1 / 2} \mathbf{H}$ и

$$
\widetilde{B}^{-1 / 2} B \widetilde{B}^{-1 / 2}=I+G,
$$


где $I$ - единичный оператор, $G$ - самосопряженный оператор Гильберта-Шмидта, а оператор $I+G$ имеет ограниченный обратный. Далее, при $i, j=1, \ldots, n$, ввиду (31),

$$
\begin{aligned}
\left\langle\left(\pi_{n} B \pi_{n}\right)^{-1} f_{i}, f_{j}\right\rangle=\langle & \left\langle\left[\pi_{n} \widetilde{B}^{1 / 2}(I+G) \widetilde{B}^{1 / 2} \pi_{n}\right]^{-1} f_{i}, f_{j}\right\rangle \\
=\frac{1}{\sqrt{\lambda_{i} \lambda_{j}}}\langle & {\left[\left(\pi_{n} \widetilde{B}^{1 / 2} \pi_{n}\right)^{-1}\left[\pi_{n} \widetilde{B}^{1 / 2}(I+G) \widetilde{B}^{1 / 2} \pi_{n}\right]\right.} \\
& \left.\left.\times\left(\pi_{n} \widetilde{B}^{1 / 2} \pi_{n}\right)^{-1}\right]^{-1} f_{i}, f_{j}\right\rangle
\end{aligned}
$$

где $\lambda_{k}$ - собственные числа оператора $\widetilde{B}: \widetilde{B} f_{k}=\lambda_{k} f_{k}, k=1,2, \ldots$. Оператор $\widetilde{B}^{1 / 2}$ в базисе $\left\{f_{n}\right\}$ имеет диагональную матрицу, поэтому

$$
\begin{aligned}
\widetilde{B}^{1 / 2} \pi_{n} \widetilde{B}^{-1 / 2} \pi_{n} & =\widetilde{B}^{-1 / 2} \pi_{n} \widetilde{B}^{1 / 2}=\pi_{n} \\
\left(\pi_{n} \widetilde{B}^{1 / 2} \pi_{n}\right)^{-1} & =\pi_{n} \widetilde{B}^{-1 / 2} \pi_{n} .
\end{aligned}
$$

Переписывая равенство (32) с учетом (33) и (34), будем иметь:

$$
\left\langle\left(\pi_{n} B \pi_{n}\right)^{-1} f_{i}, f_{j}\right\rangle=\frac{1}{\sqrt{\lambda_{i} \lambda_{j}}}\left\langle\left[\pi_{n}(I+G) \pi_{n}\right]^{-1} f_{i}, f_{j}\right\rangle .
$$

Покажем, что

$$
\left\langle\left[\pi_{n}(I+G) \pi_{n}\right]^{-1} f_{i}, f_{j}\right\rangle=\left\langle f_{i}, f_{j}\right\rangle-\left\langle\left[\pi_{n}(I+G) \pi_{n}\right]^{-1}\left(\pi_{n} G \pi_{n}\right) f_{i}, f_{j}\right\rangle .
$$

В самом деле, обозначим

$$
\left[\pi_{n}(I+G) \pi_{n}\right]^{-1} f_{i}=g_{i}, \quad i=1, \ldots, n .
$$

Тогда левая часть соотношения (36) равна $\left\langle g_{i}, f_{j}\right\rangle$, а правая, ввиду самосопряженности оператора $\pi_{n}(I+G) \pi_{n}$, принимает вид

$$
\begin{aligned}
& \left\langle\left[\pi_{n}-\left[\pi_{n}(I+G) \pi_{n}\right]^{-1}\left(\pi_{n} G \pi_{n}\right)\right] f_{i}, f_{j}\right\rangle \\
& \quad=\left\langle\left[\pi_{n}(I+G) \pi_{n}\right]\left[\pi_{n}-\left[\pi_{n}(I+G) \pi_{n}\right]^{-1}\left(\pi_{n} G \pi_{n}\right)\right] f_{i}, g_{j}\right\rangle \\
& \quad=\left\langle\pi_{n} f_{i}, g_{j}\right\rangle=\left\langle g_{i}, f_{j}\right\rangle .
\end{aligned}
$$

Рассмотрим поведение операторов $\left[\pi_{n}(I+G) \pi_{n}\right]^{-1} \pi_{n}$ при $n \rightarrow \infty$. Для этого воспользуемся известными фактами теории проекционных методов приближенного решения операторных уравнений [3], [4]. Следуя [4, гл. II, § 4], будем говорить, что обратимый оператор $A$ допускает редукцию относительно ортонормированного базиса $\left\{f_{n}\right\}$, если

$$
\forall h \in \mathbf{H}, \quad \lim _{n \rightarrow \infty}\left\|\left[\pi_{n} A \pi_{n}\right]^{-1} \pi_{n} h-A^{-1} h\right\|=0 .
$$


Известно (см. [3, гл. 4, теорема 16.4], [4, гл. II, теорема 5.1]), что для выполнения соотношения (37) для любого ортонормированного базиса необходимо и достаточно, чтобы оператор $A$ был правильным, т.е. допускал представление

$$
A=\gamma I+S+T
$$

где $\gamma=$ const $\neq 0, S$ - линейный непрерывный оператор, $\|S\|<|\gamma|$, a $T$ - линейный вполне непрерывный оператор. Ясно, что оператор $I+G$ - правильный, поэтому можно утверждать, что

$$
\forall h \in \mathbf{H}, \quad \lim _{n \rightarrow \infty}\left\|\left[\pi_{n}(I+G) \pi_{n}\right]^{-1} \pi_{n} h-(I+G)^{-1} h\right\|=0 .
$$

Следовательно, применяя теорему Банаха-Штейнгауза [14, гл. III, §4, теорема 3], будем иметь

$$
\forall n \in \mathbf{N}, \quad\left\|\left[\pi_{n}(I+G) \pi_{n}\right]^{-1} \pi_{n}\right\|<C<+\infty .
$$

Далее, ввиду (38),

$$
\begin{aligned}
& \sum_{i=1}^{n} \sum_{j=1}^{n}\left\langle\left[\pi_{n}(I+G) \pi_{n}\right]^{-1}\left(\pi_{n} G \pi_{n}\right) f_{i}, f_{j}\right\rangle^{2} \\
& \quad=\sum_{i=1}^{n}\left\|\left[\pi_{n}(I+G) \pi_{n}\right]^{-1}\left(\pi_{n} G \pi_{n}\right) f_{i}\right\|^{2} \\
& \quad \leqslant\left\|\left[\pi_{n}(I+G) \pi_{n}\right]^{-1} \pi_{n}\right\|^{2} \sum_{i=1}^{n}\left\|G f_{i}\right\|^{2} \leqslant C^{2}\|G\|_{H S}^{2},
\end{aligned}
$$

где $\|\cdot\|_{H S}$ - норма Гильберта-Шмидта. Аналогично, из неравенства $(38)$ и того, что $\left\|f_{i}\right\|=1$, получаем

$$
\left\langle\left[\pi_{n}(I+G) \pi_{n}\right]^{-1}\left(\pi_{n} G \pi_{n}\right) f_{i}, f_{i}\right\rangle \leqslant C\left\|G f_{i}\right\|,
$$

а из сходимости ряда $\|G\|_{H S}^{2}=\sum_{i=1}^{\infty}\left\|G f_{i}\right\|^{2}<+\infty$ следует, что

$$
\lim _{i \rightarrow \infty}\left\|G f_{i}\right\|=0 .
$$

Поэтому из неравенства (40) будем иметь: для любого $\varepsilon>0$ существует $N_{\varepsilon}>0$ такое, что для каждого $i=N_{\varepsilon}+1, \ldots, n$ выполняется неравенство

$$
0 \leqslant\left\langle\left[\pi_{n}(I+G) \pi_{n}\right]^{-1}\left(\pi_{n} G \pi_{n}\right) f_{i}, f_{i}\right\rangle \leqslant \varepsilon .
$$

Отсюда, выбирая $\varepsilon<\frac{1}{2}$, на основе равенств (35) и (36) получаем оценку

$$
\left\langle\left(\pi_{n} B \pi_{n}\right)^{-1} f_{i}, f_{i}\right\rangle \geqslant \frac{1}{2 \lambda_{i}}, \quad \text { когда } \quad N_{\varepsilon}+1 \leqslant i \leqslant n .
$$


Теперь легко установить справедливость свойства (2). Действительно, используя соотношения (35) и (36), обозначим:

$$
S_{n}:=\frac{1}{n^{2}}\left[n+\sum_{\substack{i=1 \\ i \neq j}}^{n} \sum_{\substack{j=1 \\ n}}^{n} \frac{\left\langle\left[\pi_{n}(I+G) \pi_{n}\right]^{-1}\left(\pi_{n} G \pi_{n}\right) f_{i}, f_{j}\right\rangle^{2}}{\left\langle\left(\pi_{n} B \pi_{n}\right)^{-1} f_{i}, f_{i}\right\rangle\left\langle\left(\pi_{n} B \pi_{n}\right)^{-1} f_{j}, f_{j}\right\rangle \lambda_{i} \lambda_{j}}\right] .
$$

Далее, на основе неравенств (39), (41) и неравенства Коши-Буняковского получаем

$$
\begin{aligned}
S_{n} \leqslant \frac{1}{n^{2}}[n & +4 \sum_{i=N_{\varepsilon}+1}^{n} \sum_{j=N_{\varepsilon}+1}^{n}\left\langle\left[\pi_{n}(I+G) \pi_{n}\right]^{-1}\left(\pi_{n} G \pi_{n}\right) f_{i}, f_{j}\right\rangle^{2} \\
& +\sum_{\substack{i=1 \\
i \neq j}}^{N_{\varepsilon}+1} \sum_{\substack{N_{\varepsilon}+1 \\
N_{\varepsilon}}} \frac{\left\langle\left(\pi_{n} B \pi_{n}\right)^{-1} f_{i}, f_{j}\right\rangle^{2}}{\left\langle\left(\pi_{n} B \pi_{n}\right)^{-1} f_{i}, f_{i}\right\rangle\left\langle\left(\pi_{n} B \pi_{n}\right)^{-1} f_{j}, f_{j}\right\rangle} \\
& \left.+2 \sum_{i=1}^{N_{j}} \sum_{j=N_{\varepsilon}+1}^{n} \frac{\left\langle\left(\pi_{n} B \pi_{n}\right)^{-1} f_{i}, f_{j}\right\rangle^{2}}{\left\langle\left(\pi_{n} B \pi_{n}\right)^{-1} f_{i}, f_{i}\right\rangle\left\langle\left(\pi_{n} B \pi_{n}\right)^{-1} f_{j}, f_{j}\right\rangle}\right] \\
\leqslant & \frac{1}{n^{2}}\left[n+4 C^{2}\|G\|_{H S}^{2}+N_{\varepsilon}\left(N_{\varepsilon}-1\right)+2\left(n-N_{\varepsilon}-1\right) N_{\varepsilon}\right] \leqslant \frac{C_{1}}{n},
\end{aligned}
$$

где $C_{1}=$ const . Следовательно, $\sum_{n=1}^{\infty} S_{n}^{2}<+\infty$. Теорема доказана.

5. Пример. Здесь мы приводим пример линейного самосопряженного положительно определенного ядерного оператора $B_{0}: \mathbf{H} \rightarrow \mathbf{H}$ и о.н.б. $\left\{f_{k}\right\} \subset \mathbf{H}$, для которых не выполняются соотношения (2) и (19). Именно,

$$
\begin{aligned}
& \varlimsup_{n \rightarrow \infty} \frac{1}{n^{2}} \sum_{i=1}^{n} \sum_{j=1}^{n} \frac{\left\langle\left(\pi_{n} B_{0} \pi_{n}\right)^{-1} f_{i}, f_{j}\right\rangle^{2}}{\left\langle\left(\pi_{n} B_{0} \pi_{n}\right)^{-1} f_{i}, f_{i}\right\rangle\left\langle\left(\pi_{n} B_{0} \pi_{n}\right)^{-1} f_{j}, f_{j}\right\rangle} \\
& \quad \geqslant \frac{1}{4}\left(\frac{\pi^{2}}{3}-3\right)=0.072467 \ldots
\end{aligned}
$$

Автор благодарит И.А. Блатова, указавшего на возможность построения примера на основе функции Грина трехточечного разностного оператора [15].

Пусть $\mathbf{H}$ - вещественное сепарабельное гильбертово пространство с о.н.б. $\left\{f_{n}\right\}_{n=1}^{\infty}$. Введем бесконечное семейство конечномерных подпространств:

$$
\mathbf{H}_{\left(2^{k}-1,2^{k+1}-2\right)}=\operatorname{span}\left\{f_{2^{k}-1}, \ldots, f_{2^{k+1}-2}\right\}, \quad k=1,2, \ldots .
$$

В каждом из этих подпространств рассмотрим конечномерные операторы

$$
B_{0}^{(k)}: \mathbf{H}_{\left(2^{k}-1,2^{k+1}-2\right)} \longrightarrow \mathbf{H}_{\left(2^{k}-1,2^{k+1}-2\right)},
$$


задаваемые в базисе $\left\{f_{n}\right\}\left(n=2^{k}-1, \ldots, 2^{k+1}-2\right)$ трехдиагональными симметрическими $\left(2^{k} \times 2^{k}\right)$-матрицами:

$$
B_{0}^{(k)}=\frac{1}{2^{2 k}}\left[\begin{array}{ccccccc}
2 & -1 & 0 & \ldots & 0 & 0 & 0 \\
-1 & 2 & -1 & \ldots & 0 & 0 & 0 \\
\ldots & \ldots & \ldots & \ldots & \ldots & \ldots & \ldots \\
0 & 0 & 0 & \ldots & -1 & 2 & -1 \\
0 & 0 & 0 & \ldots & 0 & -1 & 2
\end{array}\right], \quad k=1,2, \ldots
$$

Зададим оператор $B_{0}: \mathbf{H} \rightarrow \mathbf{H}$ в базисе $\left\{f_{n}\right\}_{n=1}^{\infty}$ с помощью блочнодиагональной бесконечной симметрической матрицы

$$
B_{0}=\operatorname{diag}\left[B_{0}^{(1)}, B_{0}^{(2)}, \ldots, B_{0}^{(k)}, \ldots\right] .
$$

Оператор $B_{0}$ ограничен, положительно определен и является ядерным. Далее, будем считать, что $n=2^{k+1}-2$, тогда

$$
\begin{aligned}
\pi_{n} B_{0} \pi_{n} & =\operatorname{diag}\left[B_{0}^{(1)}, B_{0}^{(2)}, \ldots, B_{0}^{(k)}\right] \\
\left(\pi_{n} B_{0} \pi_{n}\right)^{-1} & =\operatorname{diag}\left[\left(B_{0}^{(1)}\right)^{-1},\left(B_{0}^{(2)}\right)^{-1}, \ldots,\left(B_{0}^{(k)}\right)^{-1}\right] .
\end{aligned}
$$

Используя известные формулы обращения трехдиагональных матриц (см. [16, гл. $2, \S 2.12])$, будем иметь

$$
\left(B_{0}^{(l)}\right)^{-1}=2^{2 l}\left[\min (r, s)-\frac{r s}{2^{l}+1}\right], \quad r, s=1, \ldots, 2^{l}, \quad l=1, \ldots, k .
$$

Следовательно, при $n=2^{k+1}-2$, суммируя по блокам, получаем

$$
\begin{aligned}
\frac{1}{n^{2}} \sum_{i=1}^{n} \sum_{j=1}^{n} \frac{\left\langle\left(\pi_{n} B_{0} \pi_{n}\right)^{-1} f_{i}, f_{j}\right\rangle^{2}}{\left\langle\left(\pi_{n} B_{0} \pi_{n}\right)^{-1} f_{i}, f_{i}\right\rangle\left\langle\left(\pi_{n} B_{0} \pi_{n}\right)^{-1} f_{j}, f_{j}\right\rangle} \\
>\frac{1}{4\left(2^{k}-1\right)^{2}} \sum_{r=1}^{2^{k}} \sum_{s=1}^{2^{k}}\left[\min \left(\frac{r}{2^{k}+1}, \frac{s}{2^{k}+1}\right)-\frac{r}{2^{k}+1} \frac{s}{2^{k}+1}\right]^{2} \\
\quad \times\left[\frac{r}{2^{k}+1}\left(1-\frac{r}{2^{k}+1}\right) \frac{s}{2^{k}+1}\left(1-\frac{s}{2^{k}+1}\right)\right]^{-1} .
\end{aligned}
$$

Рассмотрим двойной интеграл (от квадрата корреляционной функции броуновского моста) как предел интегральных сумм:

$$
\begin{aligned}
& \lim _{m \rightarrow \infty} \frac{1}{m^{2}} \sum_{i=1}^{m-1} \sum_{j=1}^{m-1}\left[\min \left(\frac{i}{m}, \frac{j}{m}\right)-\frac{i}{m} \frac{j}{m}\right]^{2}\left[\frac{i}{m}\left(1-\frac{i}{m}\right) \frac{j}{m}\left(1-\frac{j}{m}\right)\right]^{-1} \\
& =\int_{0}^{1} \int_{0}^{1} \frac{[\min (x, y)-x y]^{2}}{x(1-x) y(1-y)} d x d y=\frac{\pi^{2}}{3}-3 .
\end{aligned}
$$

Учитывая это равенство и переходя к пределу в неравенстве (43), получаем соотношение (42). 


\section{СПИСОК ЛИТЕРАТУРЫ}

1. Шатских С.Я. Устойчивые эллиптически контурированные меры в гильбертовом пространстве: асимптотические свойства условных распределений. - Изв. РАЕН, сер. МММИУ, 1999, № 3, с. 43-81.

2. Михлин С. Г. Вариационные методы в математической физике. М.: Наука, 1970, $512 \mathrm{c}$

3. Красносельский М.А., Вайникко Г.М., Забрейко П.П., Рутицкий Я.Б., Стеиенко В.Я. Приближенное решение операторных уравнений. М.: Наука, 1969, $455 \mathrm{c}$.

4. Гохберг И. Ц., Фельдман И. А. Уравнения в свертках и проекционные методы их решения. М.: Наука, 1971, 352 с.

5. Скороход A. В. Интегрирование в гильбертовом пространстве. М.: Наука, 1975, $232 \mathrm{c}$.

6. Крамер Г. Математические методы статистики. М.: Мир, 1975, 648 с.

7. Ширяев А.Н. Вероятность. М.: Наука, 1989, 640 с.

8. Золотарев В. М. Одномерные устойчивые распределения. М.: Наука, 1983, $304 \mathrm{c}$.

9. Феллер В. Введение в теорию вероятностей и ее приложения. Т. 1, 2. М.: Мир, 1984,526 c., 752 c.

10. Федорюк М. В. Асимптотика: интегралы и ряды. М.: Наука, 1987, 544 с.

11. Далеикий Ю. Л., Фомин С. В. Меры и дифференциальные уравнения в бесконечномерных пространствах. М.: Наука, 1983, 384 с.

12. Богачев В. И. Гауссовские меры. М.: Наука, 1997, 352 с.

13. Гирко В. Л. Предельные теоремы для функций случайных величин. Киев: Вища школа, 1983, $208 \mathrm{c}$.

14. Люстерник Л. А., Соболев В. И. Элементы функционального анализа. М.: Наука, $1965,520 \mathrm{c}$.

15. Самарский A. A., Николаев E.C. Методы решения сеточных уравнений. М.: Наука, 1978, $591 \mathrm{c.}$

16. Ильин В. П., Кузнечов Ю.И. Трехдиагональные матрицы и их приложения. М.: Наука, 1985, 207 с.

Поступила в редакцию

8.II. 2001

Исправленный вариант

15.X.2003 